\title{
Role of particle rotations and rolling resistance in a semi-infinite particulate solid indented by a rigid flat punch
}

\author{
Antoinette Tordesillas* John Peters ${ }^{\dagger}$ \\ Maya Muthuswamy
}

(Received 11 October 2004, revised 10 March 2005)

\begin{abstract}
Particle rotations, and in particular, rolling resistance are known to have a dominant influence on the macroscopic behaviour of particulate materials. We examine the influence of these factors in the constitutive response of a semi-infinite material to indentation by a rigid flat punch on the material boundary. Extensive particle rotations
\end{abstract}

${ }^{0}$ This paper is dedicated to Professor Jim Hill on the occasion of his 60th birthday.

*Department of Mathematics and Statistics, University of Melbourne 3010, Australia. mailto: atordesi@ms. unimelb.edu.au

${ }^{\dagger}$ U. S. Army Corps of Engineers, Engineer Research and Development Center, Vicksburg, U. S. A.

${ }^{\ddagger}$ Department of Mathematics and Statistics, University of Melbourne 3010, Australia. mailto:mayadm@gmail.com

See http://anziamj.austms.org.au/V46/CTAC2004/Tord for this article, (C) Austral. Mathematical Soc. 2005. Published April 27, 2005. ISSN 1446-8735 
are found to occur near the edges of the punch where stress concentrations exist, and from where plastic strains which localise into so-called shear bands emanate. The effects of rolling resistance are found to have a significant influence on the load-deflection characteristics of the punch.

\section{Contents}

1 Introduction

2 Discrete element model

C263

3 Results and discussion

C265

4 Conclusions

$\mathrm{C} 272$

References

C273

\section{Introduction}

Determining the stresses and deformation of a semi-infinite material induced by the indentation of a rigid flat punch on the material's surface is a wellknown fundamental problem in the theories of elasticity and plasticity $[6,7]$. It is encountered in a broad range of engineering problems within soil mechanics, geophysics, industrial forming processes, biomechanics, and off-road vehicle operations, just to name a few $[9,3,10,17]$. Yet for many everyday materials (for example, geomaterials, powders, composites etc.), this problem poses complexities that are not completely understood in either elasticity or plasticity. For example, in the theory of elasticity a stress singularity occurs at the punch edge. Such a singularity cannot exist in reality: no physical body has a perfect square corner (there is always some degree of roundedness) 
and, moreover, real materials will yield at finite stress. However, although such a singularity is not a feature of real materials, it is known that an abrupt change in the contact topology occurring at the punch corner leads to regions of relatively high stress concentrations which may, in turn, initiate localised plastic deformations (that is, shear bands).

We revisit the flat punch problem for a specific class of materials known as particulate solids or granular materials. Like many other heterogeneous materials, the rich and complex behaviour of particulate solids ultimately arises from internal mechanisms acting on many length scales. For example, it is now widely accepted that particle rotations govern the macroscopic response of particulate solids to external loads. Using non-invasive experimental techniques, Oda and co-workers uncovered the role of particle rotations in shear band development in bi-axial compression tests [12]. In particular, their studies revealed that large particle rotations are concentrated inside the shear band, with relatively little or no rotations occurring outside the band. Thus a relatively high rotation gradient is generated along the boundaries of the band, a feature which has important implications in continuum micromechanical modelling $[8,11]$. In another set of experiments, Oda et al. [13] also showed that the dominant deformation mechanism at the microstructural level, which leads to extensive dilatancy in particulate solids, is inter-particle rolling and not inter-particle sliding as previously believed. This finding later motivated studies using discrete element simulations to determine the influence of rolling resistance in shear band development in biaxial compression tests. The results clearly showed that incorporating rolling resistance in discrete element simulations led to significant improvements in their predictive capabilities. Thus our specific objective is to investigate, via discrete element analysis, the role of particle rotations and rolling resistance in the response of a particulate material to indentation by a flat punch. Of specific interest is the extent to which particle rotation and rolling resistance alter both the microscopic and macroscopic response of the material to the indenting punch. We focus on two key aspects: from a microscopic level, we determine the extent of particle rotations in the region close to the punch 
face, and from a macroscopic level, we determine whether rolling resistance has any influence on the load-deflection characteristics of the punch.

The results presented here would be a useful guide for future developments in micromechanical continuum theory. Indeed, in recent years, micromechanical models associated with Cosserat (micropolar) continuum have provided a closer association with the actual media by introducing a characteristic size and enriching the continuum description with rotational degrees of freedom as well as rolling resistance $[5,15,16,18,20]$. This study can therefore assist in determining the suitability of these micromechanically-based Cosserat models in capturing the essence of particulate behaviour. The use of discrete element simulations as a virtual laboratory is particularly appropriate for the type of models developed by Tordesillas and co-workers as these models rely on the same input parameters as the DEM simulations, thereby allowing a direct comparison to be made between the discrete and continuum models.

\section{Discrete element model}

The discrete element method (DEM) [2] treats particles as distinct interacting bodies. Interactions between particles are described by contact laws that define forces and moments created by relative motions of the particles. The motion of each particle that results from the net forces and moments is obtained by integrating Newton's laws. Thus, the particles are not treated as a medium, rather, the medium behaviour emerges from the interactions of the particles comprising the assemblage [1].

The evolution of particle velocity $v_{i}$ and rotational rate $\omega_{i}$ are given by

$$
m \frac{\partial v_{i}}{\partial t}=m g_{i}+\sum_{c=1}^{N_{c}} f_{i}^{c}
$$


and

$$
I_{m} \rho \frac{\partial \omega_{i}}{\partial t}=\sum_{c=1}^{N_{c}} e_{i j k} f_{i}^{c} r_{j}^{c}+\sum_{c=1}^{N_{c}} m_{i}^{c},
$$

where $m, I_{m}$ and $\rho$ are the particle mass, moment of inertia and density respectively; $g_{i}$ is the acceleration of gravity; $f_{i}^{c}$ and $m_{i}^{c}$ the forces and moments applied at the contacts; $r_{j}^{c}$ is the radius vector linking the particle centre to the contact; $N_{c}$ is the number of contacts for the particle; and $e_{i j k}$ is the usual permutation symbol.

Two particles with radii $R_{A}$ and $R_{B}$ make contact when the distance $d$ separating the particles satisfies:

$$
d<R_{A}+R_{B} .
$$

The contact forces and moments arise from relative motion between contacting particles. The motion of each individual particle is described by the velocity of the particle centre $\dot{u}_{i}$ (or $v_{i}$ ) and the rotation about the centre. The branch vector between particle centres, $x_{i}^{A}-x_{i}^{B}$, is also the difference between the respective radii vectors that link the particle centres to the contact $r_{i}^{A}-r_{i}^{B}$. With this nomenclature, the relative motion at contact $c$ between particles $A$ and $B$ is

$$
\dot{\Delta}_{i}^{c}=\dot{u}_{i}^{A}-\dot{u}_{i}^{B}+e_{i j k}\left(r_{j}^{A} \omega_{k}^{A}-r_{j}^{B} \omega_{k}^{B}\right) .
$$

The contact moments are generated by the difference in rotations between the particles:

$$
\Delta \omega_{i}^{c}=\Delta t\left(\omega_{i}^{A}-\omega_{i}^{B}\right) .
$$

The contact forces are given by the contact laws in terms of their normal and shear components,

$$
f^{n}=\left\{\begin{array}{l}
K^{n} \Delta^{n} \\
E_{r} K^{n}\left(\Delta^{o}-\Delta^{n}\right), \quad \Delta^{n}<\Delta^{o} ;
\end{array}\right.
$$




$$
f_{i}^{s}=\left\{\begin{array}{l}
K^{s} \Delta_{i}^{s}, \\
f^{n} \tan \phi n_{i}^{s}, \quad\left|f_{i}^{s}\right| \geq f^{n} \tan \phi ;
\end{array}\right.
$$

and the contact moment

$$
m_{i}^{c}=\left\{\begin{array}{l}
K^{m} \Delta \omega_{i}^{c}, \\
f^{n} \tan \phi_{m} n_{i}^{m}, \quad\left|m_{i}^{c}\right| \geq f^{n} \tan \phi_{m} ;
\end{array}\right.
$$

where $K^{n}, K^{s}$ and $K^{m}$ are stiffness constants; $E_{r}$ is a factor to dissipate energy through stiffening the unload response; $\Delta^{n}$ and $\Delta_{i}^{s}$ are the normal and shear components of the contact displacement; $n_{i}^{s}$ and $n_{i}^{m}$ are unit vectors in the direction of the shear force and moment; $\Delta^{o}$ is the greatest value of penetration in the history of $\Delta^{n}$; and $\phi$ and $\phi_{m}$ are friction parameters. Thus note that there are two parameters $\left(K^{m}\right.$ and $\left.\phi_{m}\right)$ associated with the overall resistance to rolling at each contact.

\section{Results and discussion}

The discrete element model described in Section 2 is used to model the response of a 2D assembly of 7503 circular disks under deformation by a rigid flat punch. The disk radii ranged from 0.0333 inches to 0.05 inches, with an average radius of 0.0417 inches. The initial setup is established by dropping the particles into the box, and allowing them to settle until individual rotations/displacements became negligible. In all simulations, the punch moved down with a velocity of 0.5 inches/second for 1 second. The parameters used in the simulations are summarised in Table 1. Note that where more than one value has been used, these are listed separated by commas.

In all cases, the resulting flow pattern is not symmetric due to the random packing of the material. Figure 1 shows particle rotation rates at an indentation of 0.05 in for the cases with and without rolling resistance. Clearly, 
TABle 1: Parameters used in the simulations DEM parameters and material properties

Duration
Timestep increment
Punch velocity
Box size
Punch width
Number of particles
Value

Particle density

Smallest radius $1 \mathrm{~s}$

Largest radius

$1.843 \times 10^{-5} \mathrm{~s}$

$0.5 \mathrm{in} / \mathrm{s}$

10 in $\times 5$ in

$1,0.4$ in

7503

Average radius

$0.2476 \mathrm{lb} / \mathrm{in}^{3}$

$3.33 \times 10^{-2}$ in

Inter-particle friction coefficient $(\tan \phi)$

$5.0 \times 10^{-2}$ in

Particle-wall friction coefficient $(\tan \phi=f)$

$4.170 \times 10^{-2}$ in

Rolling friction coefficient $\left(\tan \phi_{m}\right)$

0.1

Gravity value

Energy dissipation $\left(E_{r}\right)$

$0.0,0.36$

$0.1,0.5$

$386.4 \mathrm{in} / \mathrm{s}^{2}$

Normal stiffness constant $\left(K^{n}\right)$

10

Tangential stiffness constant $\left(K^{s}\right)$

$3000 \mathrm{lbf} /$ in

Rotational stiffness constant $\left(K^{m}\right)$ $3000 \mathrm{lbf} /$ in

$0,50,300,1000 \mathrm{lbf} / \mathrm{rad}$ 
Scale: $[\mathrm{rad} / \mathrm{s}]$

\begin{tabular}{ll}
\hline & \\
\hline 0 & 4.9
\end{tabular}

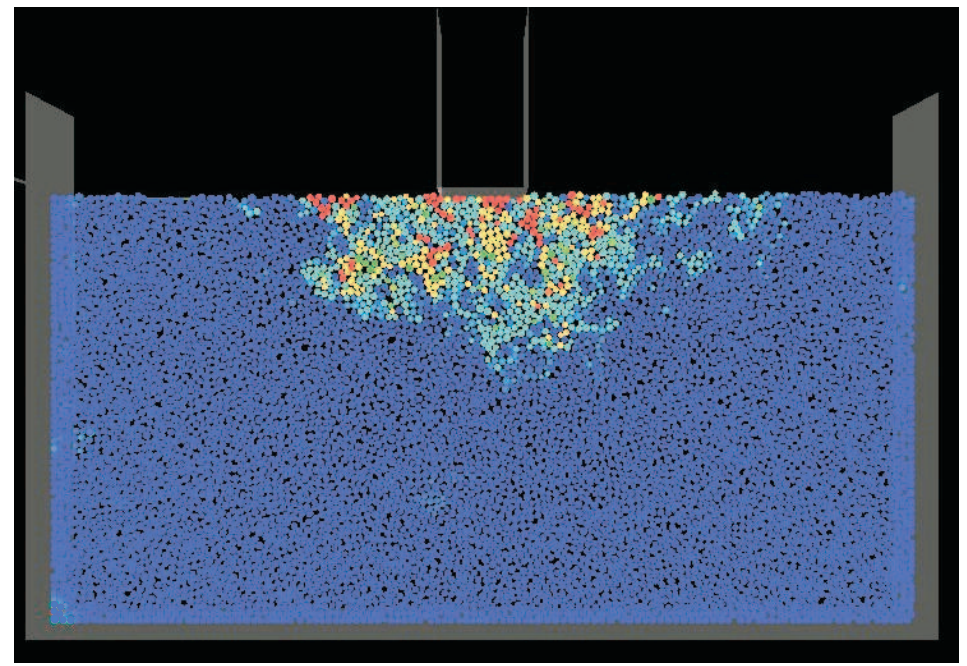

(a)

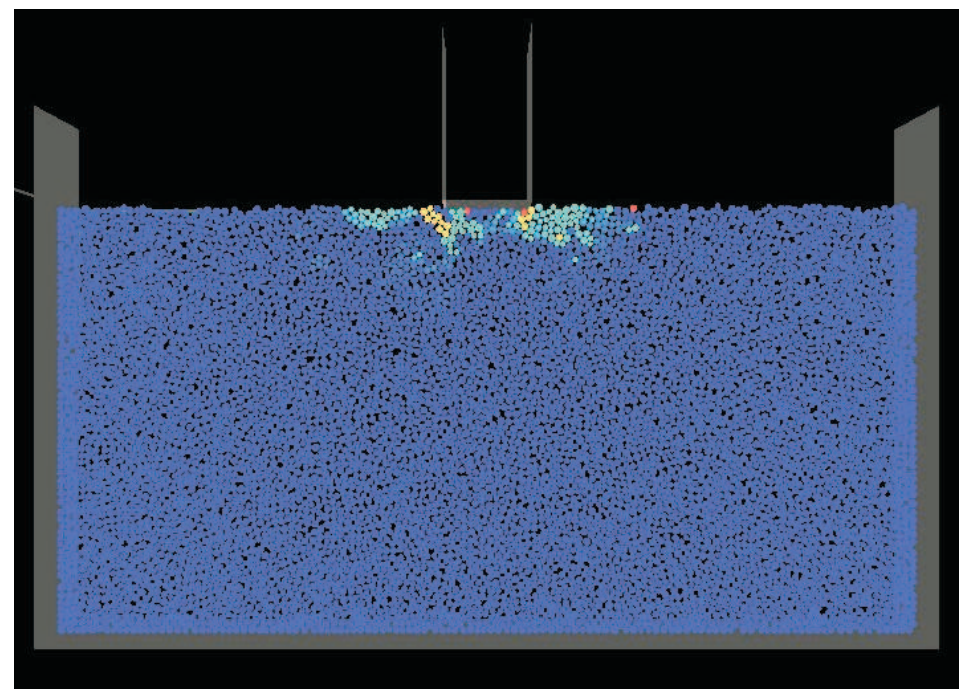

(b)

Figure 1: Particle rotation rate at 0.05 in indentation $(f=0$, punch width $=1 \mathrm{in}, \tan \phi_{m}=0.1$ ): (a) $K^{m}=0 \mathrm{lbf} / \mathrm{rad}$, (b) $K^{m}=50 \mathrm{lbf} / \mathrm{rad}$. 
Scale: [rad]

2.5

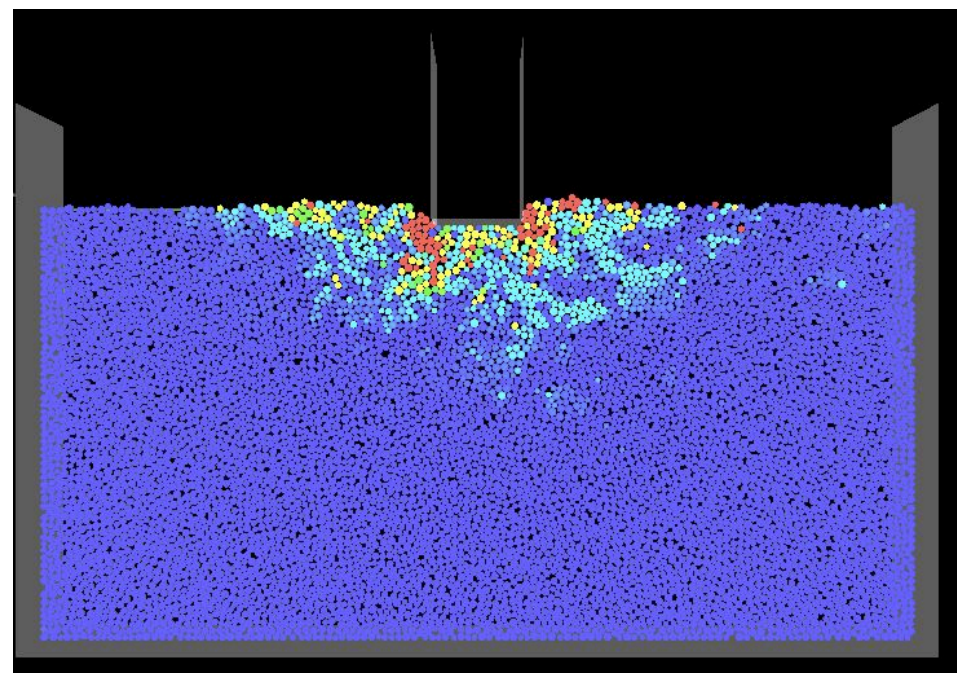

Figure 2: Particle rotation at 0.25 in indentation $(f=0$, punch width $=$ $\left.1 \mathrm{in}, K^{m}=300 \mathrm{lbf} / \mathrm{rad}, \tan \phi_{m}=0.1\right)$. Download the movie.

without any rolling resistance (a) the rotations are large and unrealistic. Introducing rolling resistance (b) into the simulation certainly improves the resulting rotations underneath the punch. In Figure 2, we show particle rotations at an indentation of 0.25 in [14].

Note that the rotation pattern shown in Figure 2 is consistent with the rotations derived from a finite element simulation based on a recently developed micropolar model using the same parameters and level of indentation [19], as shown in Figure 3-note that only half of the domain is shown due to symmetry. Also note that a finer mesh (that is, darker region) is used for the region directly underneath the punch. 


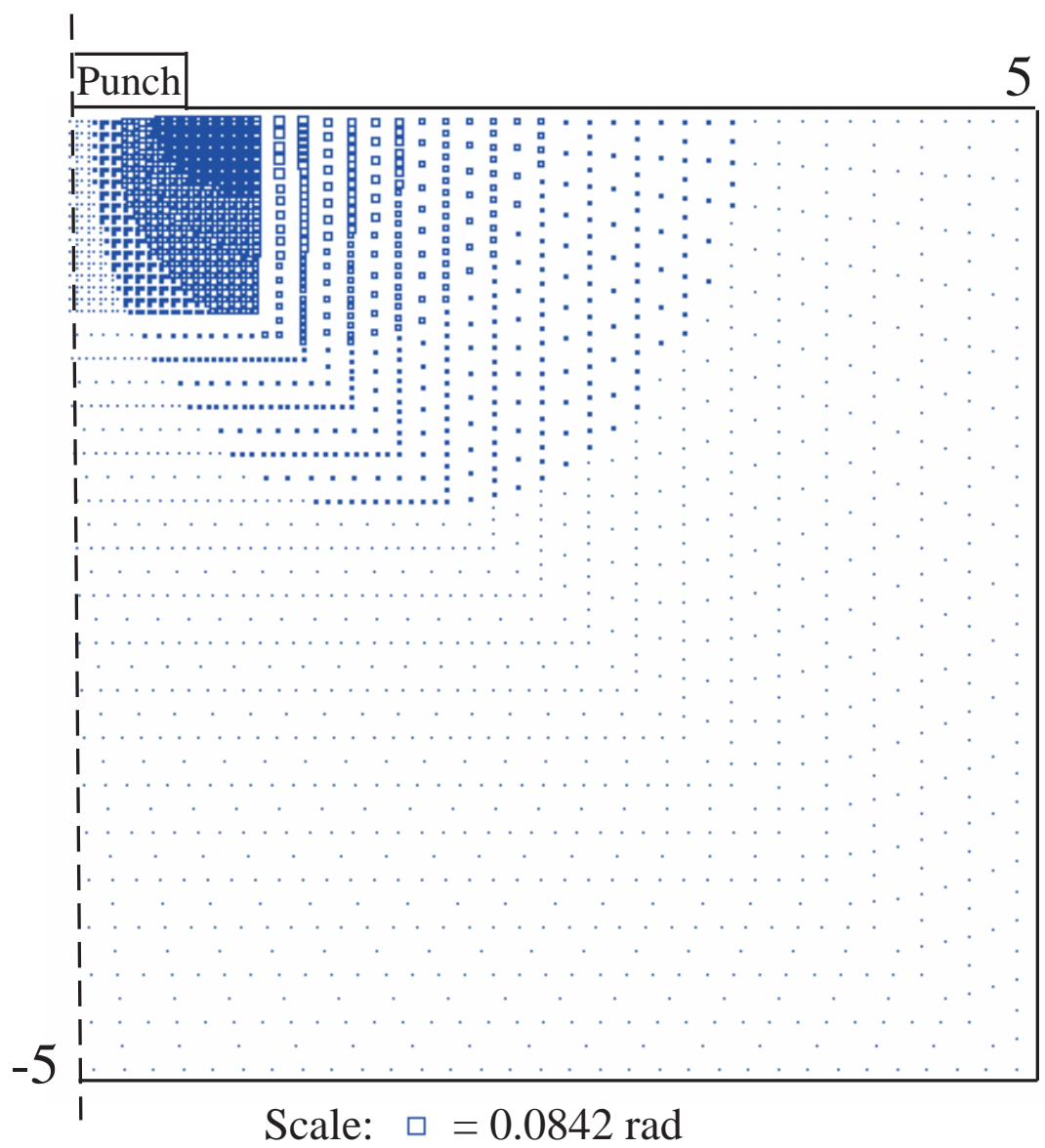

FiguRE 3: FEM rotations for half-space at 0.25 in indentation $(f=0$, punch width $\left.=0.5 \mathrm{in}, K^{m}=300 \mathrm{lbf} / \mathrm{rad}, K^{s}=3000 \mathrm{lbf} / \mathrm{in}, K^{n}=3000 \mathrm{lbf} / \mathrm{in}\right)$. 
TABLE 2: Key for Figure 4.

\begin{tabular}{|l|cccc|}
\hline Name & $f$ & Punch width (in) & $K^{m}(\mathrm{lbf} / \mathrm{rad})$ & $\tan \phi_{m}$ \\
\hline $\mathrm{Km}=0$ & 0 & 1 & 0 & 0.1 \\
$\mathrm{Km}=50$ & 0 & 1 & 50 & 0.1 \\
$\mathrm{Km}=300$ & 0 & 1 & 300 & 0.1 \\
$\mathrm{Km}=1000$ & 0 & 1 & 1000 & 0.1 \\
Frictional & 0.36 & 1 & 50 & 0.1 \\
Small & 0 & 0.4 & 50 & 0.1 \\
Mtan=0.5 & 0 & 1 & 50 & 0.5 \\
\hline
\end{tabular}

In general, we see that large rotations are generated near the punch corners where high stress concentrations exist. These rotations, in turn, lead to dilatation in the regions adjacent to the sides of the punch. Directly below the punch (recall Figure 2) we see a region (near triangular in shape) in which there are no rotations. This is in keeping with the well-known and experimentally observed "quasi-solid" region beneath the punch, which moves with the punch in rigid body motion.

Throughout deformation, rotations are a dominant deformation mechanism at the microstructural (particle) level.

Figure 4 shows the total force on the punch (that is, the load) for displacements of up to 0.475 in with the specific parameters used given in Table 2. Varying the rolling resistance produced marked differences in the load-displacement characteristics.

With all other parameters kept the same, we find that the total indentation load required to reach the same displacement increased slightly as the average radius decreased (or the punch width increased). This inverse relationship, albeit weak, is consistent with the micropolar model for 3-dimensional polydisperse granular media developed by Gardiner and Tordesillas [4]. Similarly, changing the coefficient of friction at the contact interface while keeping all other parameters constant only leads to very small changes in the total 


\section{Load-displacement graph}

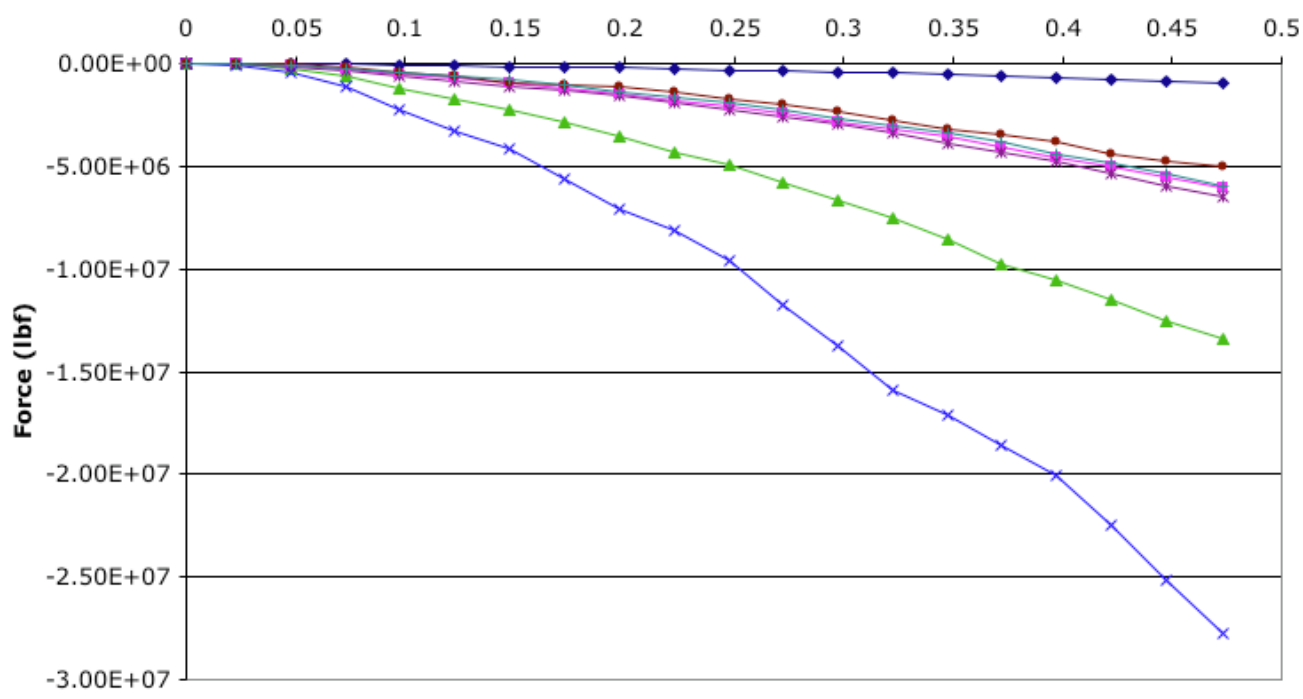

Displacement (in)

$\bullet-\mathrm{Km}=0 \rightarrow-\mathrm{Km}=50 \rightarrow-\mathrm{Km}=300 \rightarrow \mathrm{Km}=1000 \rightarrow$ Frictional $\rightarrow$ Small $\rightarrow$ Mtan $=0.5$

FIGURE 4: Load-displacement graph for punch 
load required to indent the material to the same depth. The same applies for the coefficient of rolling friction, which indicates that the rotational stiffness constant, equation (8), is the dominant contributor to rolling resistance.

\section{Conclusions}

It is widely agreed that particle rotations do play a key role in deformation of granular assemblies, and we have shown this for the rigid flat punch problem. However, simply incorporating particle rotations is not enough-resistance to particle rolling must also be included due to its significant influence on the constitutive response of the material at both the microscopic and macroscopic level.

We also examined the influence of other factors, such as the average radius of particles and the coefficient of friction at the contact interface between the punch and the particulate material. Our findings indicate that these factors have only a minor influence on the constitutive response of the material to the punch, when compared with rotations and rolling resistance. These suggest that models of granular materials need to incorporate at least particle rotations and rolling resistance, before they can provide a reasonably accurate representation of real particulate behaviour.

Acknowledgments: The support of the US Army Research Office through a grant to AT (grant number DAAD19-02-1-0216) is gratefully acknowledged, as is the University of Melbourne Advanced Research Computing section through a grant of computer time. 


\section{References}

[1] P. A. Cundall. A discrete future for numerical modeling. Discrete Element Methods: Numerical Modeling of Discontinua, ASCE Geotechnical Special Publication No. 117, 3-4, 2002. C263

[2] P. A. Cundall, O. D. L. Strack. A discrete numerical model for granular assemblies. Geotechnique, 29:47-65, 1979. C263

[3] W. Ehlers. Foundations of multiphasic and porous materials. In W. Ehlers, J. Bluhm, editors, Porous media - Theory, experiments and numerical applications, Springer-Verlag, Berlin, 2002. C261

[4] B. Gardiner, A. Tordesillas. The effects of particle size distribution based on a homogenisation scheme for high-resolution analysis of three-dimensional micropolar media. Powder Technology, in review. $\mathrm{C} 270$

[5] B. Gardiner, A. Tordesillas. Micromechanics of shear bands. International Journal of Solids and Structures, 41:5885-5901, 2004. http://dx.doi.org/10.1016/j.ijsolstr.2004.05.051 C263

[6] G. M. L. Gladwell. Contact Problems in the Classical Theory of Elasticity, Sijthoff and Noordhoff, The Netherlands, 1980. C261

[7] R. Hill. The Mathematical Theory of Plasticity, Oxford University Press, London, 1950. C261

[8] K. Iwashita, M. Oda. Micro-deformation mechanism of shear banding process based on modified distinct element method. Powder Technology, 109:192-205, 2000. http://dx.doi.org/10.1016/S0032-5910(99)00236-3 C262

[9] K. L. Johnson. Contact Mechanics. Cambridge University Press, 1985. $\mathrm{C} 261$ 
[10] Y. Liu, A. E. Kerdok, R. D. Howe. A nonlinear finite element model of soft tissue indentation. ISMS, 67-76, 2004. C261

[11] M. Oda, K. Iwashita. Study on couple stress and shear band development in granular media based on numerical simulation analyses. International Journal of Engineering Science, 38:1713-1740, 2000. http://dx.doi.org/10.1016/S0020-7225(99)00132-9 C262

[12] M. Oda, H. Kazama. Micro-structure of shear band and its relation to the mechanism of dilatancy and failure of granular soils. Geotechnique, 48(4):465-481, 1998. C262

[13] M. Oda, J. Konishi, S. Nemat-Nasser. Experimental micro-mechanical evaluation of strength of granular materials: effect of particle rolling. Mechanics of Materials 1, 267-283, 1982. http://dx.doi.org/10.1016/0167-6636(82)90027-8 C262

[14] Particle Visualisation Software User Manual, University of Melbourne Software Engineering Department, 2003. C268

[15] A. Tordesillas, J. Peters, B. Gardiner. Insights on 1D localisation theory and micromechanical constitutive laws. Geotechnique, 54:1-4, 2004. http://dx.doi.org/10.1680/geot.54.5.327.46727 C263

[16] A. Tordesillas, J. Peters, B. Gardiner. Shear band evolution and accumulated microstructural development in Cosserat media. International Journal for Numerical and Analytical Methods in Geomechanics, 28:981-1010, 2004. http://dx.doi.org/10.1002/nag.343 C263

[17] A. Tordesillas, J. Shi. Frictional indentation of dilatant granular materials. Proceedings of the Royal Society of London. Series A: Mathematical, Physical and Engineering Sciences, 455 (1981): 261-283, 1999. C261 
[18] A. Tordesillas, S. Walsh. Incorporating rolling resistance and contact anisotropy in micromechanical models of granular media. Powder Technology, 124(1-2):106-111, 2002. http://dx.doi.org/10.1016/S0032-5910(01)00490-9 C263

[19] S. D. C. Walsh, A. Tordesillas. Finite element methods for micropolar models of granular materials. Applied Mathematical Modelling, submitted. C268

[20] S. D. C. Walsh, A. Tordesillas. A thermomechanical approach to the development of micropolar constitutive models for granular media. Acta Mechanica, 167(3-4):145-169, 2004. http://dx.doi.org/10.1007/s00707-003-0072-z C263 